\title{
Le terminologue et le spécialiste de domaine
}

John Humbley

\section{(2) OpenEdition}

Journals

Édition électronique

URL : http://journals.openedition.org/asp/2789

DOI : 10.4000/asp.2789

ISSN : 2108-6354

\section{Éditeur}

Groupe d'étude et de recherche en anglais de spécialité

\section{Édition imprimée}

Date de publication : 1 décembre 1998

Pagination : 137-149

ISSN : 1246-8185

\section{Référence électronique}

John Humbley, «Le terminologue et le spécialiste de domaine », ASp [En ligne], 19-22 | 1998, mis en ligne le 09 mars 2012, consulté le 03 mai 2019. URL : http://journals.openedition.org/asp/2789 ; DOI : 10.4000/asp.2789

Ce document a été généré automatiquement le 3 mai 2019.

Tous droits réservés 


\title{
Le terminologue et le spécialiste de domaine
}

\author{
John Humbley
}

\section{L'enseignement des langues de spécialité : comment faire?}

1 Le point fort du colloque GERAS de Limoges en 1997 était le lien entre le monde du travail et les langues, et plus particulièrement les technologies qui renforcent ce lien. La réflexion qui suit se place en amont : l'apport de la terminologie à l'enseignement des langues de spécialité. Cette réflexion ne se veut pas plaidoyer pro domo, et encore moins incitation à transformer les cours de langue de spécialité en cours de terminologie. Il s'agit bien plutôt d'une interrogation sur les liens qui existent, ou qui pourraient exister, entre la spécialité ou future spécialité de l'étudiant et sa formation linguistique. La terminologie constitue, de ce point de vue, le pont entre la profession et la pratique des langues.

On peut, par ailleurs, placer cette réflexion dans la perspective des changements qui s'annoncent au niveau de l'organisation de la vie universitaire en France. Nous allons être confrontés, nous autres professeurs de langues, à des changements venus d'en haut (proposés par le ministère) et d'en bas (poussés par les étudiants, par la société) et si nous n'avons pas quelques idées claires sur ce que nous souhaitons, nous risquons de nous voir imposer des réformes mal conçues. Tout changement n'est pas négatif. Parmi les changements que nous avons appelés de nos voeux, nous mettons en première ligne les échanges d'étudiants et d'enseignants, dans le cadre des programmes Erasmus, Socrates et autres Tempus. En ce qui concerne les étudiants, notre démarche vise à les accompagner dans leurs études, qui se feront dans une autre langue et dans un autre pays. Pour les enseignants, il s'agit d'échanges également, non seulement des professeurs de langues mais aussi des enseignants des matières de spécialité. C'est donc une occasion à saisir.

Parmi les changements qui risquent de nous être imposés, on pense à la réforme du CAPES, qui serait vidé de son contenu littéraire et civilisationniste. Si on peut être 
sceptique quant au bien-fondé de cette élimination radicale, il serait temps de définir la place qui revient à l'enseignement des langues de spécialité dans la formation de maîtres, linguistes ou non. On peut également espérer que les conditions de recrutement pour le supérieur seront refondues et que les jeunes qui ont fait des recherches poussées dans les langues de spécialité (toutes langues vivantes confondues d'ailleurs) pourront accéder aux fonctions d'enseignants-chercheurs dans de bonnes conditions.

\section{Quelques définitions}

Certains termes posent problème... dont la terminologie. La confusion qui entoure le sujet même de cet exposé s'explique assez facilement. D'un point de vue assez superficiel, elle est due au sens qu'on lui prête dans la langue de tous les jours : lorsqu'on veut minimiser une affaire on dit qu'il s'agit d'un simple problème de terminologie. La terminologie, dans les pays francophones, fait partie de la politique linguistique de défense de la langue, préoccupation absente de la terminologie d'Europe Centrale. La terminologie était peu connue par ailleurs en France, parce qu'absente du cursus, mais la situation a bien changé depuis une dizaine d'années, grâce au travail accompli à Rennes 2, à Lyon 2, à Paris 3, et depuis longtemps par les écoles d'interprètes et de traducteurs. Les malentendus sont donc parfois dus à un manque d'information. Mais la raison la plus importante de cette confusion est à chercher dans la terminologie et dans son histoire.

5 Nous savons que la terminologie telle que nous la connaissons aujourd'hui doit son statut de «discipline interdisciplinaire » à l'ingénieur autrichien Eugen Wüster. Wüster lance dès le début des années 1930 un programme très ambitieux d'unification des sciences grâce à une structuration conceptuelle universelle dont la terminologie représente la clé de voûte. Mettant l'accent sur l'organisation conceptuelle des choses, la terminologie wüstérienne tend à faire abstraction des problèmes proprement linguistiques. Elle trouve son expression la plus typique sous la forme de la normalisation industrielle et des systèmes d'organisation des connaissances de type documentaire.

6 Cette conception par trop philosophique de la terminologie a fait l'objet de bien des critiques, qui font valoir que la terminologie n'a pas toujours les moyens de ses ambitions. Ces critiques ne sont pas sans fondement, même si certains détracteurs ont un peu tendance à « jeter le bébé conceptuel avec l'eau du bain philosophique ». On reproche à la terminologie de ne pas tenir suffisamment compte des aspects linguistiques de la langue de spécialité, de s'enfermer dans une vision trop cloisonnée de la division entre domaines ou spécialités, et d'avoir une vision trop idéaliste de ce qui constitue le concept, pièce maitresse de la structure. Lorsqu'on fait le tour de toutes les critiques dont fait l'objet la terminologie appelée parfois dominante, on peut se demander s'il reste encore quelque chose à récupérer.

7 On peut citer à cet égard l'avis d'un sceptique notoire, Juan Carlos Sager, qui dit en introduction de son manuel :

This book denies the independent status of terminology as a discipline but affirms its value as a subject in almost every contemporary teaching programme. There is no substantial body of literature which could support the proclamation of terminology as a separate discipline and there is not likely to be. Everything of import than can be said about terminology is more appropriately said in the context of linguistics or information science or computational linguistics. (1990:1) 

la terminologie, qui ne serait plus une formation en tant que telle, mais une méthode, une méthodologie qui entrerait dans la composition de toutes sortes de formation, formation de linguistes, bien entendu, mais aussi de spécialistes de différents domaines, surtout lorsqu'il s'agit d'une formation plurilingue.

En quoi consiste actuellement la terminologie ? Si on se rapporte encore à Sager, la réponse est très ciblée :

Terminology is now associated generally with the provision of information services which require the collection of information about terms in order to compile dictionaries and glossaries, and more recently entries in term banks. (op cit : 4)

voit bien l'intérêt d'incorporer une formation de terminologue dans celle d'un traducteur technique, mais qu'en est-il de celle d'un futur juriste, chimiste ou biologiste? Là on a intérêt à s'inspirer de la division du travail proposée par Daniel Gouadec (1990 : 4) entre terminologue, le généraliste, terminographe, qui rédige les fiches, et terminoticien, qui garantit leur informatisation. Je plaiderais très fermement pour une conception très large du mandat du terminologue, qui devrait comporter non seulement les aspects lexicographiques, mais ceux préconisés par Wüster, revus et corrigés à la lumière des acquis des soixante dernières années. Le terminologue est un intermédiaire, spécialiste de communication, interface entre les spécialités, les sciences cognitives, la documentation et l'expression linguistique.

11 Comme l'exprime Heribert Picht :

Das übergeordnete Ziel der Terminologie ist es, die Fachkommikation zu erleichern und zu fördern und damit den fachlichen Wissenstransfer zu sichern (1997 : 33)

Ce n'est pas un hasard si la plus grande manifestation européenne de terminologie est le congrès de la société pour la terminologie et le transfert des connaissances, et c'est dans ce sens-là que nous souhaitons orienter notre discussion à propos de la terminologie.

13 Toujours en matière de définition, on peut dire, en ce qui concerne les langues de spécialité, qu'il existe un accord plus général que pour la terminologie. On songe à la définition consensuelle que Pierre Lerat a donnée lors de l'ouverture du congrès du GERAS de 1997. Le trait distinctif serait la visée pragmatique de la communication, visée qui peut être très différente d'une situation à une autre. Il s'ensuit que les langues de spécialité représentent des entités très différentes, très diversifiées selon notamment les différentes disciplines auxquelles elles s'attachent. Ce n'est pas une découverte pour le GERAS de constater que les problèmes linguistiques des juristes, des économistes, des scientifiques, des techniciens sont très différents, et il faut se méfier de toute solution globale. Nous aborderons surtout les domaines tertiaires, mais sans oublier les sciences dures, même appliquées.

\section{Que peut le terminologue?}

14 Si nous acceptons la réalité du terminologue protéiforme, son rôle dans l'enseignement supérieur et, à terme, secondaire serait d'assurer le lien entre les langues et l'enseignement spécialisé. Ceci implique une double compétence de la part du terminologue, compétence linguistique et compétence dans le domaine. Cette double compétence n'a rien de nouveau. M. Bacquet la préconisait il y a déjà plus de vingt ans dans le cadre des LEA alors tout juste sorties des cartons du Ministère de l'Éducation 
nationale. Il y a néanmoins une différence. Bacquet recommandait que le linguiste suive une formation poussée dans la spécialité, pour en devenir en quelque sorte un expert luimême. Ceci n'est pas le but du terminologue. Chacun conserve sa sphère de compétence, des sphères qui se recoupent largement tout en restant spécifiques. On peut citer le cas de la terminologue Zélie Guével, aujourd'hui professeur à l'Université Laval à Québec, mais pendant de longues années terminologue à l'ordre des comptables du Québec. Interrogée sur ses compétences en comptabilité, elle insiste toujours sur le fait qu'elle n'est pas devenue comptable, mais elle est capable de dialoguer avec les comptables, de leur poser des questions et de comprendre les réponses, de traduire n'importe quel texte de comptabilité. Ceci me semble être ce qu'on peut exiger du terminologue surtout dans le contexte de la formation.

C'est donc le terminologue qui prend contact avec les enseignants de la spécialité, qui domine le programme d'enseignement, et qui établit le programme linguistique. C'est le terminologue qui prend contact avec le spécialiste étranger venu faire des cours de spécialité dans le domaine, et facilite la communication.

Si c'est le terminologue qui prend cette initiative, c'est parce que la terminologie prend comme point de départ la réalité extralinguistique et les problèmes de sa présentation, de sa représentation, monolingue et surtout bilingue et, nous l'espérons plurilingue.

\section{Exemples}

Nous sommes donc à la recherche de pistes pour concrétiser ces propositions. Je voudrais présenter trois ou quatre exemples de travail concret dans des domaines de spécialité, plutôt que des exemples de collaboration réussie entre terminologue et spécialiste de domaine, non pas que cela n'existe pas, mais parce qu'il faut chercher des initiatives plus originales.

\subsection{Droit : droit d'auteur et copyright}

18 Cet exemple, qui relève du domaine du droit, est atypique à plusieurs points de vue. Atypique, car directement lié à un travail de lexicographie et atypique, car sans lien avec la pédagogie. Ce qui nous permet de le retenir, c'est justement la collaboration entre juriste et terminologue qui, à nos yeux, pourrait être transférée à une situation d'enseignement.

L'origine de la collaboration est un projet juridique d'un intérêt très général. Nous savons que les accords internationaux sur la libéralisation du secteur tertiaire butent depuis très longtemps sur les épineux problèmes de droits d'auteur. Ces problèmes sont particulièrement compliqués. D'une part, il s'agit de protéger les droits légitimes des auteurs de différents ordres, et au-delà de protéger le patrimoine culturel, mais d'autre part, il s'agit aussi, très concrètement, de comprendre les législations nationales et internationales très complexes et souvent incompatibles. Voilà pourquoi droit d'auteur n'égale pas copyright - il s'agit de deux concepts juridiques différents. Il faut donc d'abord voir clair pour pouvoir prendre des mesures efficaces. C'est dans cet esprit qu'une équipe de juristes Droit des systèmes d'information et de communication (le DISC) du Centre d'études sur la coopération juridique internationale, sous l'impulsion d'Isabelle de Lamberterie, a décidé de faire une liste des termes du domaine du droit d'auteur/ 
copyright, et d'élaborer des définitions. Elle a réuni des juristes spécialistes du droit français, belge, anglais, américain, canadien et international, qui ont d'abord fait une liste des termes. Ensuite ils ont commencé à remplir des fiches, qu'elle avait conçues selon un modèle terminologique classique. C'est au moment de les collationner et de les comparer que l'équipe de coordination s'est rendu compte qu'il y avait un problème: les fiches étaient si complètes, si compliquées, qu'il était difficile pour les juristes non pas de les comprendre, mais de les présenter de façon claire aux non-juristes qui doivent s'occuper de droit d'auteur sur le plan international. C'est alors qu'une terminologue, Véronique Alory, est intervenue. Sa première démarche était d'informatiser les fiches déjà existantes (c'est la fonction terminoticienne déjà mentionnée), ce qui a nécessité une restructuration, et puis elle a commencé le travail de comparaison des concepts. Cette mise à plat s'est faite à l'aide de grilles très simples, mais très systématiques, qui donnent un aperçu synoptique, et qui permettent d'établir des comparaisons lorsqu'il est possible, et de constater des lacunes. Pour la fiche artiste interprète, par exemple, la lecture des cinq fiches disponibles permet de constater que pour le droit français, un artiste interprète est un comédien, un chanteur, un musicien, un danseur, un artiste de variétés ou un artiste de cirque, soit une définition par extension. Il en est de même pour les droits belge et anglais, mais pour le droit américain c'est un musicien, mais pas un comédien ni un danseur ni un artiste de cirque. Par ailleurs, la fiche ne permet pas de savoir si les chanteurs et les artistes de variétés sont compris.

Face à cette présentation de leur réalité, la réaction des juristes était mitigée ; ils étaient contents d'avoir un outil de travail, mais réservés quant à l'idée de se servir des grilles pour la présentation aux non-juristes. Quoi qu'il en soit, le travail sur le dictionnaire se poursuit, cet exemple de collaboration est prometteur.

21 Pour en savoir plus sur les qualifications de la terminologue il suffit de signaler qu'elle a une formation de linguiste (surtout allemand et russe) complétée par le DESS du CRIM ou Centre de recherche sur l'ingénierie multilingue de l'INaLCO, mais pas de formation juridique. Elle avait fait un audit sur les problèmes de communication commerciale à l'EDF (Alory 1995), ce qui a dû la préparer au dialogue interdisciplinaire. Il me semble qu'une personne comme Véronique Alory pourrait rendre service à tout l'enseignement des langues dans une faculté de droit, où les problèmes de communication internationale se situent autant sur le plan des différences de systèmes juridiques que sur les expressions proprement linguistiques. Mais c'est un chapitre que nous n'aborderons pas ici.

\subsection{Banque}

Les deux exemples que j'aimerais présenter maintenant sont applicables et d'ailleurs déjà appliqués dans le cadre des LEA. Contrairement aux étudiants non linguistes, les LEA ont une maigre formation disciplinaire. Si les enseignements de langue ne renforcent pas les "matières d'application ", les étudiants arrivent gravement handicapés sur le marché du travail. Le point fort des diplômés LEA doit être leur capacité à être opérationnels en plusieurs langues dans des situations professionnelles. Les exemples suivants sont puisés dans le cursus de Paris 13, dont le LEA est spécialisé, dès la première année, dans les professions de la banque et des assurances. systématique de la terminologie qui permet d'assimiler l'essentiel de la matière 
d'application dans les trois langues du cursus. Si l'on commence par examiner le concept chèque (fig. 1), on peut partir du schéma publié par Picht. Deux commentaires: pédagogique et terminologique.

Le schéma peut servir de point de départ à l'étude... en allemand... du chèque, complété par des documents de différentes sortes : « realia » pris dans des banques suisses, articles de presse, etc. La partie terminologique ne représente donc qu'une étape dans la démarche, qui doit être complétée par différentes «mises en discours», et éventuellement d'autres schémas terminologiques (par exemple un dictionnaire des acteurs et des actions du chèque, qui mettent en jeu les verbes, exclus du premier schéma). Après avoir complété l'étude en allemand, on procède à la recherche d'équivalents en français, et on puise dans le cours de banque dispensé par un banquier (fig. 2). Cette partie de l'exercice est indispensable. Rien de plus frustrant que d'apprendre dans une langue étrangère sans apparier directement les équivalents et sans faire le point dans la langue maternelle, qui doit rester la langue dominante. La troisième phase est la transposition dans une autre langue étrangère, par exemple, l'anglais, de la même façon, en s'appuyant sur les «realia » et éventuellement des articles, afin de compléter le schéma en anglais.

\section{Figure 1}

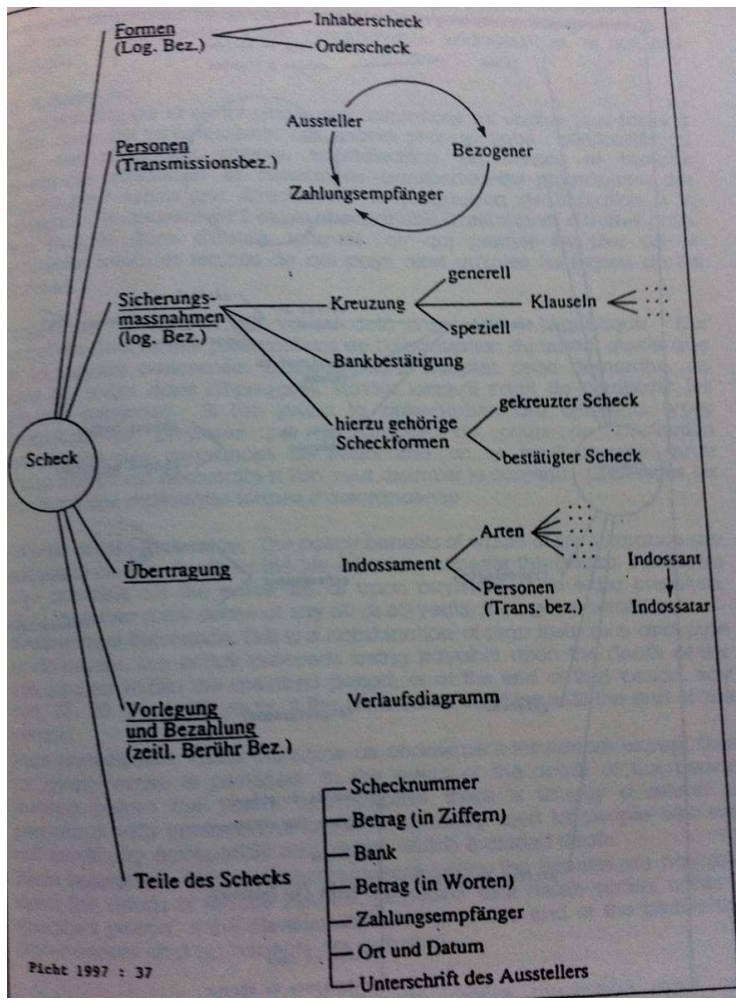

Le schéma permet de balayer l'ensemble du système, car on sait qu'il n'est pas possible de trouver tous les termes dans les documents qu'on peut présenter et qu'il faut compléter. 


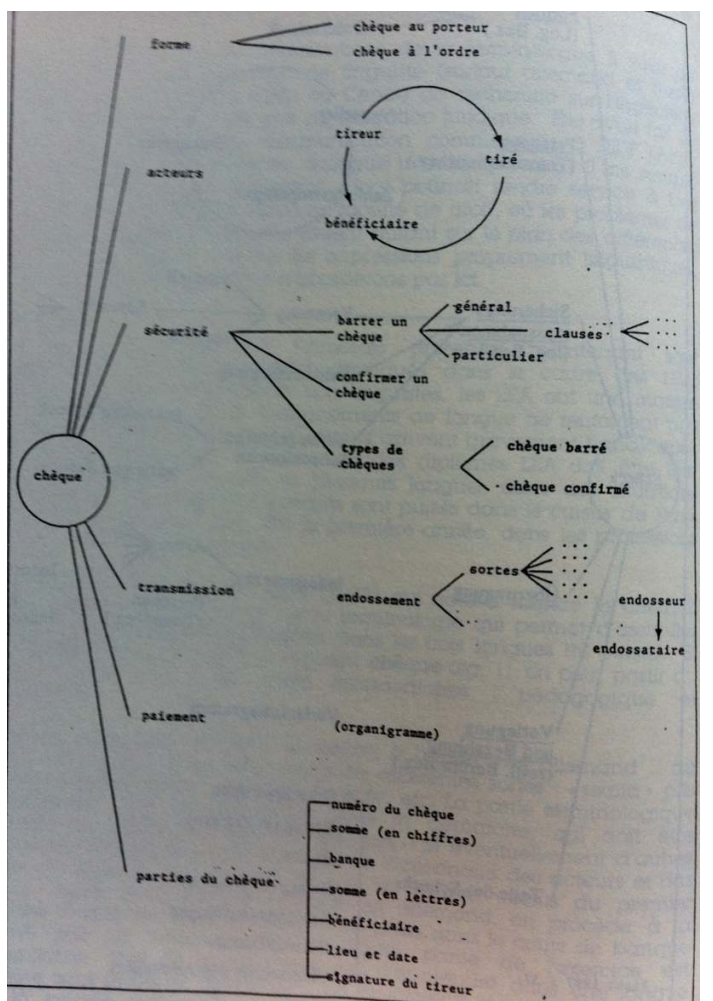

D'un point de vue terminologique, on constate que ce schéma intègre les relations classiques de générique/spécifique, de tout et partie, ainsi que d'autres types de relations moins souvent invoquées. D'un point de vue logique, ce schéma est une représentation "impure ", mais il est pratique. On a donc quelques séries génériques: les types de chèques, les types de mesures de sécurité; on a au moins une série partitive: les différentes parties d'un chèque; on a des relations de transmission, des relations de processus, donc aussi temporelles, représentées par des organigrammes. Il s'agit donc d'une représentation pragmatique, pédagogique, et non pas théorique.

\subsection{Assurances}

Le programme de la terminologie des assurances est encore plus facile à établir avec les professionnels, car encore plus appliqué : généralités au cours des premières années, spécialisation en licence et maîtrise (assurances maritimes et assurances transports). Le programme des langues peut suivre très directement la progression de l'initiation à la profession. Heureusement il existe des manuels d'assurances d'autres pays, donc rédigés dans d'autres langues, ce qui permet en plus de se familiariser avec les réalités de ces pays ainsi qu'avec les façons de les exprimer.

L'apport de la terminologie va au-delà d'un soutien linguistique. Elle permet de clarifier des présentations de l'organisation du travail quelle que soit la langue concernée. D'ailleurs, sans adopter cette démarche, on risque de rester dans l'à-peu-près, surtout lorsqu'il s'agit de comparer les différents systèmes. Si l'on prend la présentation des différents types d'assurance-vie proposés par le support de cours de l'Institution australienne des assurances de Melbourne, on se rend compte qu'un certain travail est 
nécessaire si l'on veut assimiler le contenu. Ci-dessous les définitions des différentes formes d'assurance-vie :

1. Whole of Life Insurance. The policy benefits of whole of life insurance are payable on the death of the life insured whenever this occurs. Premiums are payable for the entire life, or upon payment of an extra premium. [sic] Premium may cease at say 60 or 65 years, the age of retirement.

2. Endowment Insurance. This is a combination of term insurance and pure endowment, the policy proceeds being payable upon the death of the life insured within the specified period, or at the end of that period, say ten, 15, 20, 25 or 30 years, if the life insured survives until the end of the period.

3. Pure endowment. This is the same as endowment insurances except that no death cover is provided. In the event of the death of the person insured before the policy term expires there is usually a refund of premiums with interest. This cover is normally used for people who are not medically acceptable for a cover which included death.

4. Term Insurance. Under a term insurance policy the benefits are payable upon the death of the life insured, provided that death occurs within a specified period. If the life insured survives to the end ot the period the cover ceases and no benefits are payable.

Afin d'y voir clair, on extrait chaque critère mentionné dans ces « définitions » afin de pouvoir les comparer. Cette comparaison peut prendre la forme d'une grille d'analyse où on note les critères retenus pour distinguer les différentes catégories.

Figure 3. Life insurance

\begin{tabular}{|l|c|c|c|}
\hline & death cover & death in set period & insured = beneficiary \\
\hline \hline term insurance & + & + & - \\
\hline whole of life insurance & + & - & - \\
\hline pure endowment insurance & - & & + \\
\hline endowment insurance & + & - & + \\
\hline
\end{tabular}

Nous établissons les critères à partir de la dernière définition : term insurance. On apprend d'abord que l'indemnisation intervient au décès de la «tête assurée ", critère que nous avons intitulé death cover, et que nous pouvons indiquer comme présent, en apposant un +. Il est précisé toutefois que le décès doit impérativement intervenir pendant la période couverte par le contrat, critère que nous avons décrit comme death within set period, et coché comme applicable. La dernière partie de la citation ajoute l'information suivante : l'assuré ne peut être bénéficiaire de cette couverture : ou il décède avant (auquel cas un tiers est bénéficiaire) ou il survit, et... « no benefits are available ».

Le reste du tableau est rempli selon la même méthode. Il serait possible d'ajouter d'autres critères, comme celui du paiement de la prime, mais puisque les trois critères initialement retenus suffisent pour bien distinguer les quatre types d'assurance-vie, on peut en profiter pour faire la distinction entre critères discriminatoires et informations encyclopédiques. 

une langue étrangère: il est nécessaire de bien ancrer l'ensemble dans la langue maternelle, donc retour au cours des professionnels, ou recherche dans la documentation des compagnies d'assurance. On apprend ainsi que «term insurance» correspond à assurance en cas de décès, "pure endowment » à assurance en cas de vie (ce qui donc ne relève pas strictement de l'assurance vie mais plutôt de la capitalisation), ainsi de suite. Et puis on peut tenter le même jeu dans d'autres langues étrangères du cursus. À partir de ces tableaux, les étudiants peuvent formuler eux-mêmes des définitions. Comme les étudiants finissent souvent par reconnaître : tant qu'on n'est pas capable de définir un concept du domaine, on ne l'a pas vraiment compris et encore moins assimilé.

\subsection{Mécanique de la rupture}

Jusqu'ici les exemples sont tirés du secteur tertiaire, et on peut se demander si les sciences dures et les technologies se prêtent à ce genre de traitement. La question reste effectivement ouverte, mais on peut verser au dossier au moins une expérience

jeune doctorante, Skora Setti, vient de terminer une thèse sur la terminologie (trilingue) de la mécanique de la rupture. C'est par la terminologie qu'on se rend compte de la diglossie qui règne dans tous les secteurs de la recherche scientifique et technologique, la tension qui existe entre l'anglais, langue de communication et de publication internationales, et le français, ou l'allemand ou d'autres langues au sein du laboratoire et dans la salle de cours. Mais grâce à cette initiation à une terminologie spécifique, la doctorante a appris à dialoguer avec les spécialistes. Elle en avait besoin pour ses recherches, mais elle sait renvoyer l'ascenseur. Un des produits secondaires, le glossaire trilingue, rend service dans le laboratoire ; en outre, elle est désormais capable de corriger la prose anglaise des spécialistes, car elle le fait en connaissance de cause.

Comment a-t-elle fait? D'abord, et à un niveau très simple, en utilisant les méthodes de terminologie afin de systématiser les informations souvent éparses. À partir d'une définition dûment estampillée par les autorités du domaine, elle arrive à en dériver d'autres, comme déformation plastique dérivée de déformation élastique. À un niveau plus avancé, Skora Setti s'est rendu compte qu'une partie des difficultés conceptuelles ressenties par les spécialistes eux-mêmes s'explique du fait que le domaine de la mécanique de la rupture est en fait constitué de plusieurs domaines plus vastes, dont les notions cohabitent de façon variable, selon le point de vue adopté par le spécialiste. C'est ainsi qu'une même notion peut avoir une certaine définition si elle est considérée sous l'angle de la fatigue, et une autre définition si le point de vue est celui de la mécanique de la rupture. Ces points de vue sont non seulement légitimes, mais probablement nécessaires à ce nouveau domaine, mais ils peuvent créer des confusions, surtout dans l'esprit des étudiants. À ces difficultés s'ajoutent celles de la diglossie qui caractérise tant de domaines scientifiques et techniques, et qui compliquent encore ces différences d'interprétation. Skora Setti décrit ces phénomènes dans des termes de parasynonymie, c'est-à-dire d'une façon qui relève davantage de l'analyse linguistique que de la pédagogie d'une langue de spécialité, mais il s'agit pour l'instant d'une thèse. La prochaine étape est justement l'adaptation didactique, et j'espère qu'elle aura l'occasion de tirer un bénéfice pédagogique des connaissances du domaine, et de la méthode qu'elle a durement acquise au cours de sa longue initiation à la recherche en terminologie. 


\section{Portrait du terminologue?}

Les quatre exemples que nous venons de citer ne constituent pas le portrait-robot du parfait terminologue du département de langues de spécialité, tant s'en faut. Mais il me semble qu'ils indiquent des pistes que nous pourrions explorer ensemble, et avec les spécialistes des domaines concernés. En effet, si nous adoptons une conception assez large de la terminologie, qui ne se limite pas aux activités de terminographie, elle pourra jouer le rôle d'intermédiaire, d'interface, entre linguistes et spécialiste, rôle qui est appelé à prendre de l'importance compte tenu de la multiplication des échanges à tous les niveaux. En outre, cette conception de la terminologie n'est pas du tout nouvelle, elle est tout à fait conforme aux orientations définies par son fondateur, si on veut bien examiner, et réinterpréter ses écrits.

Mais qui dit présence d'un terminologue dans un département, ou présence de la terminologie dans un enseignement, dit formation des formateurs? On a souvent constaté que le frein à la généralisation de l'enseignement de la terminologie est la pénurie des enseignants. Il faut donc former les formateurs. Là des progrès considérables ont été enregistrés, en formation initiale, et peut-être encore plus dans les Universités d'automne de terminologie de Rennes 2 . Il reste encore à réfléchir comment incorporer des éléments de terminologie dans la formation disciplinaire, mais même là il existe des ouvertures, des spécialistes convaincus du bien-fondé de cet aspect de la formation. Nous avons eu l'occasion de constater que les activités de terminologie en mathématiques se faisaient surtout dans un contexte pédagogique, et les exemples peuvent être multipliés dans d'autres domaines. Serait-il utopique de vouloir instiller une approche terminologique dans les programmes des écoles normales? Une suggestion allant dans ce sens a déjà été faite en Belgique (Schaetzen 1992). Une formation plus poussée semble indispensable pour les professeurs de langues de spécialité, dans le supérieur, bien entendu, mais aussi dans le secondaire, ce qui pose le problème plus général de cette formation. Quel est le rôle du GERAS dans ces formations? Et comment convaincre les différents $\mathrm{CNU}$ du bien-fondé de la formation non seulement terminologique, mais spécialisée aussi lorsqu'il s'agit de qualifier les candidats à des postes de langagiers dans les facultés de sciences ou les écoles d'ingénieurs? À notre avis, l'argumentaire doit être construit à partir de travaux de recherche de qualité indiscutable, suivis d'applications sur le terrain d'utilité tout aussi indiscutable.

\section{BIBLIOGRAPHIE}

Alory, Véronique. 1995. «Terminologie commerciale : concrétisation d'une approche terminologique à Electricité de France ». Terminologies nouvelles 13, 28-34.

Australian Insurance Institute. 1994. Affiliate General Programme: Elements of law and insurance. Melbourne : AII. 
Gouadec, Daniel. 1990. Terminologie. Constitution des données. Paris : AFNOR.

Lerat, Pierre. 1997. « Approches linguistiques des langues spécialisées ». ASp 15-18, 1-10.

Picht, Heribert. 1997. « Erarbeitung und Anwendung begrifflicher Strukturierungen ». Hermes (Handelshojskolen i Aarhus) 18, 33-50.

Rey, Alain. 1992 [1979]. Terminologie : noms et notions. Coll. Que sais-je ? Paris : Presses Universitaires de France.

Sager, Juan Carlos. 1990. A Practical Course in Terminology Processing. Amsterdam/Philadelphie : John Benjamin.

Setti, Skora. 1997. «La relation concept-objet autour des définitions de termes ». Thèse de doctorat, Université Paris.

Schaetzen, Caroline de. 1992. « La barrière du vocabulaire en première candidature de sciences ». La Banque des mots 43, 15-50.

\section{RÉSUMÉS}

La terminologie, conçue comme méthode interdisciplinaire visant à transmettre des connaissances par des moyens linguistiques, est appelée à jouer un rôle important dans l'enseignement des langues de spécialité. En prenant comme point de départ les concepts de la formation professionnelle des étudiants, la terminologie assure le lien entre discipline, langue maternelle et langues vivantes. Les possibilités de cette démarche sont illustrées dans les domaines du droit, de la mécanique de la rupture, et des professions de la banque et des assurances.

Terminology, understood as an interdisciplinary method aiming at transmitting knowledge by means of language, should play an important part in the teaching of LSP. Taking the contents of students' professional training as a starting point, terminology is the link between the subject field, mother tongue and foreign languages. The possibilities of this method are illustrated in the fields of law, banking, insurance and fracture mechanics.

\section{INDEX}

Keywords : banking, insurance, law, LSP, professional training, stress mechanics, teaching, terminology

Mots-clés : assurance, banque, droit, enseignement, formation professionnelle, langue de spécialité, mécanique de la rupture, terminologie

\section{AUTEUR}

\section{JOHN HUMBLEY}

John Humbley est professeur à l'Université Paris 13 - Villetaneuse, où il intervient dans les formations de LEA et de sciences du langage et chercheur au LLI - INaLF CNRS. Il est par ailleurs responsable du Centre de terminologie et de néologie, centre de ressources qui participe à la réalisation d'un réseau européen de centres de documentation terminologique. À ce titre, il participe à la publication de revues de terminologie (entre autres, le numéro spécial de 
Terminologies nouvelles sur la documentation et la terminologie) et de lexicologie.

humbley@eila.univ-paris-diderot.fr 